\title{
VARIACIÓN ESPACIAL DE ÍNDICES ESPECTRALES SOBRE ÁREAS QUEMADAS EN SUDAMÉRICA
}

\author{
S. OPAZO SALDIVIA ${ }^{(1)}$ y F. RODRÍGUEZ-VERDÚ( ${ }^{(2)}$
}

(1) Escuela de Ciencia Agropecuaria (Universidad de Magallanes) Avda. Bulnes, 01855, Punta Arenas (Chile)

(2) Departamento de Geografía (Universidad de Alcalá) C/ Colegios, 2, Alcalá de Henares (Madrid) 28002

Correo electrónico de contacto: sergio.opazo@umag.cl

RESUMEN. El presente trabajo se orienta al análisis de la variación en la respuesta espectral que presentan las áreas quemadas de distintas regiones de Sudamérica. La extracción de información espectral se ha realizado usando imágenes MODIS (MCD43), sobre perímetros de áreas quemadas extraídos previamente mediante interpretación visual de imágenes de mayor resolución espacial (Landsat y CBERS). El estudio ha utilizado dos índices de área quemada (NBR y BAI) y variables auxiliares de vegetación y clima.

Los resultados muestran que las variables de vegetación explican en mejor forma las variaciones que presentan los índices en las distintas zonas quemadas. En general, se observó que los grupos que corresponden a zonas con mayores niveles de arbolado muestran los valores más elevados en los índices de áreas quemadas.

ABSTRACT. The present work is directed to the analysis of the variation of the spectral response shown in burnt areas of different South-America regions. The obtaining of the spectral information has been done using MODIS images (MCD43), within burnt area perimeters previously extracted by means of visual interpretation of higher spatial resolution images (Landsat and CBERS). The study has used two burnt area indices (NBR and BAI) and auxiliary information of vegetation and climate.

Results shown that vegetation variables explain in a better way the indices variations in the different burnt areas. In general, it was observed that groups within more forested areas shown the highest values in the burnt area indices.

Palabras clave: variación de la respuesta espectral, áreas quemadas, MODIS, índices NBR y BAI, Sudamérica. 
Key-Words: spectral response variation, burnt land areas, MODIS, NBR and BAI Indexes, South America.

Enviado el 7 de julio de 2007 Aceptado el 10 de octubre de 2007

\section{Introducción}

Cada año grandes extensiones de superficies son afectadas por el fuego en todos los continentes. La mayoría de estos incendios son causados por el hombre normalmente por actividades derivadas de labores agrícolas, mantenimiento de pastizales, desbroce del terreno para la minería o urbanización, aunque también ocurren producto de fenómenos naturales, principalmente a causa de los rayos (Moreno, 1998; FAO, 2006).

La pérdida de valiosos recursos naturales, el incremento de los gases de efecto invernadero en la atmósfera, el efecto sobre los ciclos biogeoquímicos e hidrológicos y el aumento en la erosión son algunas de las razones por lo que los incendios son considerados como una de las principales causas del cambio climático (Levine, 1996; Puigdefábregas y Mendizabal, 1998; van der Werf et al., 2004).

Para comprender mejor el impacto de los incendios forestales sobre la vegetación, su efecto en las reservas de carbono y su rol en el funcionamiento de los ecosistemas es necesario conocer sus patrones de comportamiento a escala global (Tansey et al., 2004).

En la actualidad son numerosos los satélites que orbitan la tierra proveyendo imágenes a diferentes escalas que permiten cuantificar de manera bastante precisa la superficie afectada por los incendios. Utilizando imágenes de baja resolución espacial se han generado diversas iniciativas para cartografiar área quemada a escala global (Barbosa et al., 1999; Justice et al., 2002; Simon et al., 2004; Tansey et al., 2004; Roy et al., 2005).

Una de las metodologías más utilizadas para la discriminación de áreas quemadas es la comparación multi-temporal entre imágenes pre- y post-incendio. Dado que el efecto causado por el fuego tiene su principal impacto en las superficies vegetales, se han utilizando frecuentemente algunos índices de vegetación, lo que permite realzar el análisis temporal de este elemento.

Otra línea que se ha utilizado en la estimación de áreas quemadas es la generación de índices espectrales especialmente adaptados a la señal del carbón, lo que permite una menor confusión con superficies de comportamiento similar, facilitando su discriminación y reduciendo los errores de comisión. En este ámbito, los índices más utilizados en los últimos años y que han ofrecido buenos resultados han sido el NBR (Normalized Burned Ratio) (Key y Benson, 1999) y el BAI (Burn Area Index) (Martín, 1998); asimismo, algunos autores han obtenido resultados satisfactorios con otros índices como el GEMI, MIRBI y SAVI (Pereira, 1999; Trigg y Flasse, 2001; Chuvieco et al., 2002).

Independientemente del índice utilizado, la señal quemada o respuesta espectral de las áreas quemadas es variable por muchos motivos, algunos de ellos son el tiempo entre 
la quema y la captura de la imagen, la humedad del terreno o la época del año, aunque el factor más relevante parece ser el tipo de ecosistema. Algunas validaciones realizadas para cartografías de área quemada a gran escala han mostrado grandes diferencias dependiendo del tipo de vegetación afectada (Sifakis et al., 2004; Parisien et al., 2006; Chuvieco et al., 2007; Loboda et al., 2007).

Normalmente para definir el umbral de diferenciación entre quemado y no quemado se utiliza información obtenida de zonas quemadas previamente identificadas, de ellas se extrae un valor que es utilizado para la obtención de los perímetros quemados, o bien de los píxeles semillas que posteriormente son la base para la obtención de la cartografía en los algoritmos de 2 fases. Este valor es difícilmente generalizable especialmente cuando se trata de zonas muy extensas con gran diversidad de vegetación, induciendo a errores en la precisión de la cartografía.

En este sentido, el presente estudio tiene como objetivo analizar la variabilidad en la respuesta de diferentes zonas quemadas utilizando índices especialmente adaptados a su señal, relacionándolos con otras variables auxiliares que permitan conocer en mejor medida el comportamiento de ellas de cara a definir criterios para el establecimiento de umbrales flexibles que se adapten a las condiciones regionales, disminuyendo así los errores en la estimación global.

\section{Antecedentes}

La Red Latinoamericana de Teledetección e Incendios Forestales (RedLaTIF) ha llevado a cabo un proyecto de cartografía de áreas quemadas en Sudamérica para el año 2004 (AQL2004); una de las cuestiones más discutidas en dicho estudio fue el establecimiento de los umbrales, dado que la gran extensión de terreno abarcada incluía todo tipo de ecosistemas, desde zonas desérticas hasta cerrados bosques tropicales. El umbral utilizado finalmente se ajustó para que todos los tipos de ecosistemas quedasen incluidos y no se omitiesen áreas quemadas. Esto generó resultados disímiles para los distintos ecosistemas.

El presente estudio utiliza los perímetros de alta resolución recopilados para la validación de AQL2004. Estos perímetros fueron obtenidos a partir de la interpretación visual de imágenes satelitales de mayor detalle espacial (Landsat $30 \mathrm{~m}$ y CBERS $20 \mathrm{~m}$ ) que cubren gran parte de los ecosistemas de Sudamérica, con ellos se han realizado extracciones sobre imágenes MODIS con el fin de conocer el comportamiento espectral de las áreas quemadas en las distintas regiones del continente.

\section{3. Área de estudio}

El área de estudio comprende los diferentes perímetros quemados extraídos previamente sobre imágenes satelitales de mayor detalle espacial y que cubren gran parte de los ecosistemas de Sudamérica. Cada uno de ellos ha sido utilizado para la extracción de la información espectral (Figura 1). 


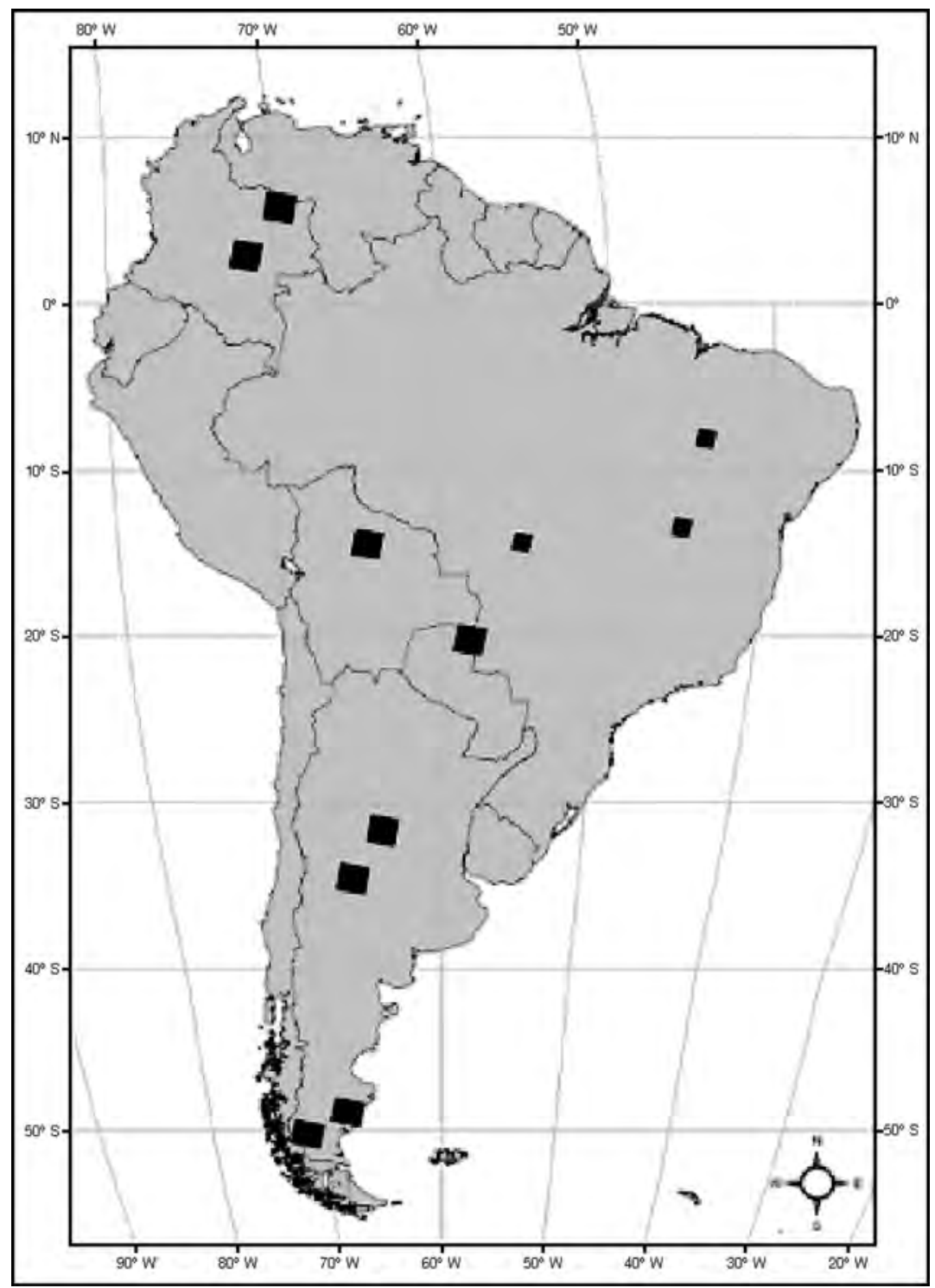

Figura 1. Ventanas utilizadas para la extracción de los perímetros de áreas quemadas

\section{Metodología}

\subsection{Variables auxiliares}

Para analizar la variabilidad que presentan las distintas zonas quemadas se han utilizado como variables auxiliares la clasificación climática según Köppen (Beck et 
al., 2006), el mapa de tipos de vegetación realizado a partir de imágenes MODIS (MODQ12) categorizada con la leyenda del IGBP (International Geosphere - Biosphere Programme) y la componente de arbolado de los campos continuos de vegetación (Vegetation Continuos Fields), esta última variable es realizada por el Departamento de Geografía de la Universidad de Maryland a partir de datos MODIS y entrega la proporción (\%) de arbolado (Figura 2).

\subsection{Extracción de información espectral sobre imágenes de satélite}

La extracción de la información espectral se realizó sobre imágenes MODIS del año 2004, específicamente se utilizó el producto estándar MCD43 (http://edcdaac.usgs. gov/modis/mcd43b4v5.asp), que ofrece datos corregidos del efecto provocado por el ángulo de visión del sensor y de la influencia atmosférica. Los datos cuentan con siete canales espectrales con una resolución espacial de $1.000 \mathrm{~m}$. y se entregan convertidos en valores de reflectividad (Tabla 1).

Tabla 1. Bandas producto MODIS MCD43

\begin{tabular}{|c|c|}
\hline Banda & Ancho de banda $(\mathrm{nm})$ \\
\hline 1 & $620-670$ \\
\hline 2 & $841-876$ \\
\hline 3 & $459-479$ \\
\hline 4 & $545-565$ \\
\hline 5 & $1230-1250$ \\
\hline 6 & $1628-1652$ \\
\hline 7 & $2105-2155$ \\
\hline
\end{tabular}

Sobre las zonas quemadas identificadas previamente se realizó un muestreo aleatorio, considerando una densidad mínima de puntos por cada perímetro ( 2 muestras/ha.); de esta forma los polígonos de mayor tamaño cuentan con mayor número de muestras (Figura 3). De cada punto se extrajo la información entregada por los siete canales de la imagen MODIS y la información entregada por las variables auxiliares. 


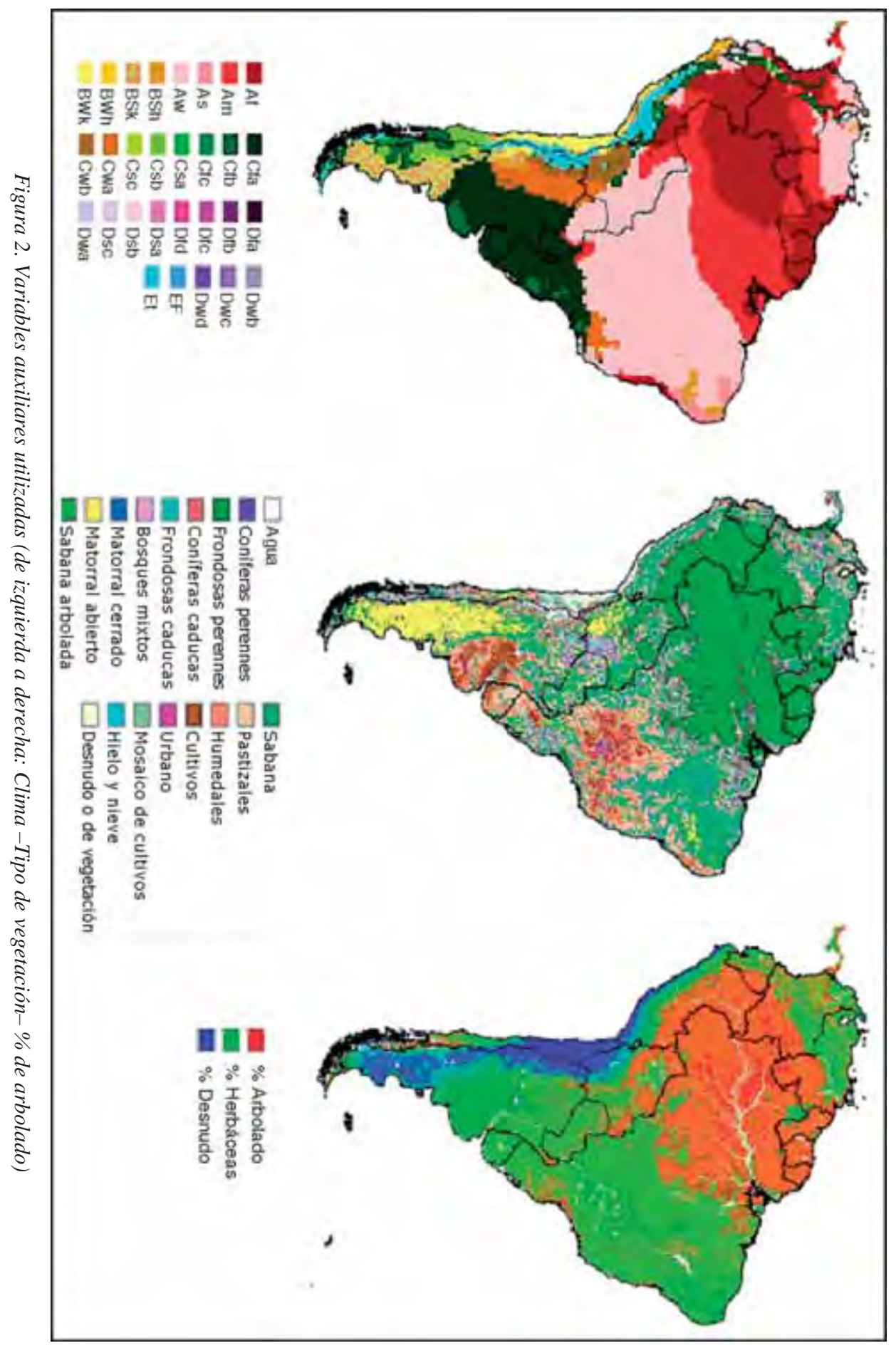




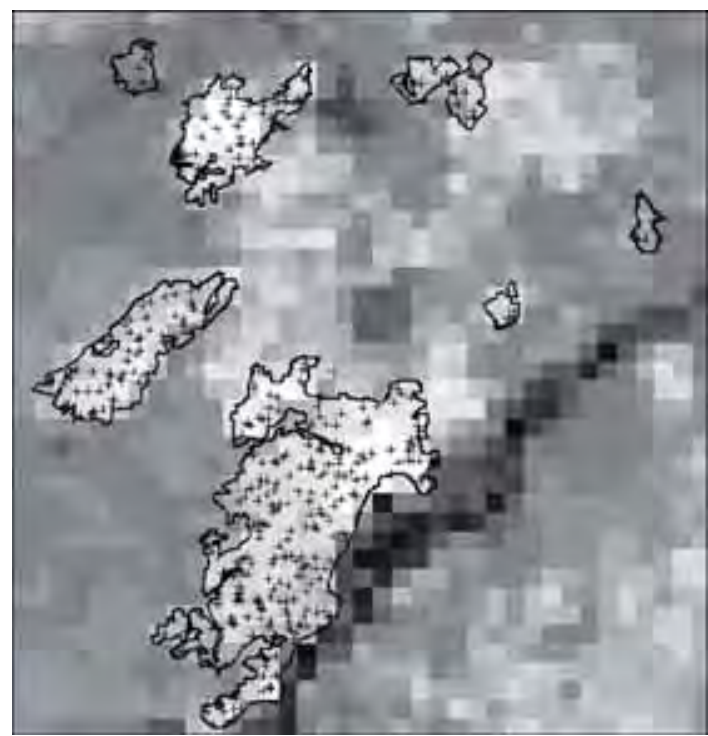

Figura 3. Muestreo aleatorio para la extracción de información espectral de áreas quemadas

\section{3. Índices espectrales}

Con la información espectral obtenida se procedió al cálculo de los índices espectrales especialmente adaptados a la señal quemada, en este caso se utilizaron el NBR y el BAIm. Ambos índices han mostrado buenos resultados en la generación de cartografía de área quemada (Key y Benson, 1999; Chuvieco et al., 2002; Martín et al., 2002; Heredia et al., 2003; Cocke et al., 2005; Miller, 2007).

El NBR fue definido por Key y Benson (1999) como:

$$
N B R=\frac{\rho N I R-\rho S W I R}{\rho N I R+\rho S W I R}
$$

Para nuestro caso, utilizando el producto MCD43, se define $\rho N I R$ y $\rho S W I R$ como la reflectividad en la banda 2 y la banda 7 , respectivamente.

El BAI fue definido por Martin (1998) específicamente para discriminar el área afectada por el fuego sobre imágenes NOAA - AVHRR (Advanced Very High Resolution Radiometer). Martín et al. (2002) realizaron una modificación para el BAI insertando información proveniente del SWIR en lugar de la correspondiente al canal rojo, esto favoreció su capacidad discriminatoria ya que esta región espectral es más propicia para la cartografía de áreas quemadas que la banda del rojo. Este nuevo índice resultó definido como:

$$
B A I m=\frac{1}{(p c S W I R-\rho S W I R)^{2}+(p c N I R-\rho N I R)^{2}}
$$


Siendo $\rho c S W I R$ y $\rho c N I R$ las reflectividades de un punto de convergencia para áreas quemadas en el SWIR e infrarrojo cercano; para el producto MCD43, $\rho S W I R$ y $\rho N I R$ son la reflectividad en las bandas 7 y 2 . Los nuevos puntos de convergencia definidos fueron 0,2 para el infrarrojo cercano $(\rho c N I R)$ y 0,08 para el $(\rho c S W I R)$.

\subsection{Análisis estadístico}

Con el propósito de estudiar el gran rango de variación que presentan los índices espectrales en las diferentes zonas muestreadas hemos propuesto la utilización de tres fuentes auxiliares, dos de ellas orientadas a la variable vegetación y una climática. Para el análisis se consideró en primer lugar el cálculo de estadísticas descriptivas (frecuencia, media, desviación típica) para los índices espectrales y las variables auxiliares. Posteriormente, para la aplicación de las pruebas estadísticas y dada la alta desviación que presenta el índice BAI, fue necesaria su normalización (BAIn) utilizando el logaritmo neperiano para su transformación (Sokal y Rholf, 1995).

A continuación, de cara a conocer el comportamiento de los índices espectrales en relación a las distintas variables auxiliares se realizó un análisis de comparación de medias (ANOVA). En el caso de la variable de tipos de vegetación se probó también agrupar la variable en grupos más generales, obteniéndose 3 clases: arbustivas, matorrales y pastizales. Para facilitar el análisis de comparación con la variable de porcentaje de arbolado se realizó la clasificación de la misma utilizando como punto de corte el percentil $10 \%$.

\section{Resultados}

\subsection{Estadísticas descriptivas}

A continuación podemos apreciar el histograma de frecuencias de los índices espectrales (Figura 4), vemos que los valores de los índices tienen un comportamiento normal; además, se entrega una Tabla (2) con las principales estadísticas.
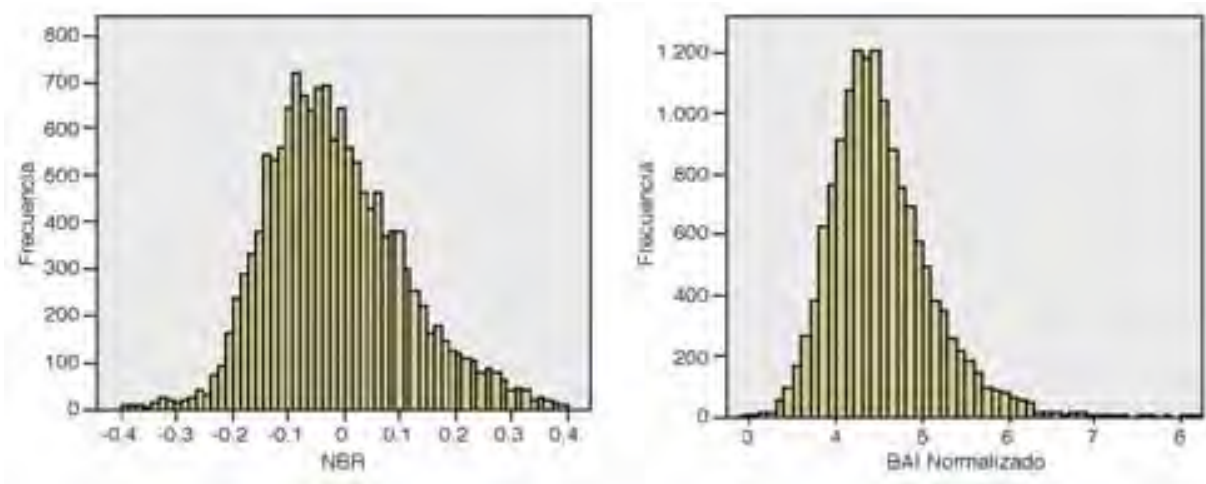

Figura 4. Histograma de frecuencias de los índices NBR y BAIn 
Tabla 2. Estadísticas descriptivas

\begin{tabular}{|c|c|c|c|c|c|}
\cline { 2 - 6 } \multicolumn{1}{c|}{} & $\mathrm{N}$ & Mínimo & Máximo & Media & Desviación típica \\
\hline NBR & 14469 & $-0,39$ & 0,40 & $-0,0162$ & 0,12201 \\
\hline BAIn & 14469 & 2,97 & 9,67 & 4,52 & 0,58 \\
\hline
\end{tabular}

En los siguientes gráficos se observa el comportamiento de las variables auxiliares de clima, tipo de vegetación y porcentaje de arbolado (Figuras 5, 6 y 7). Como vemos en la variable climática los climas ecuatoriales concentran la mayoría de los datos $(93,2 \%)$, esto se debe a que en estas zonas se presentan las condiciones propicias para que los incendios alcancen grandes dimensiones, por lo que se ha obtenido un número mayor de muestras en estas áreas. Por el contrario, en el tipo climático de estepa fría los incendios son escasos y no alcanzan dimensiones tan amplias por esto el número de muestras es menor. Por su parte los tipos de vegetación con mayor número de muestras son 9 y 10 (herbáceas), seguidos de 7 (matorral abierto) y 8 (sabana arbolada), debido a que normalmente este tipo de combustible facilita en mayor medida la propagación de los incendios. En cuanto al porcentaje de arbolado se aprecia que los valores tienen una media en torno a $22 \%$ y siendo escasos los píxeles con una cobertura de arbolado mayor al $50 \%$.

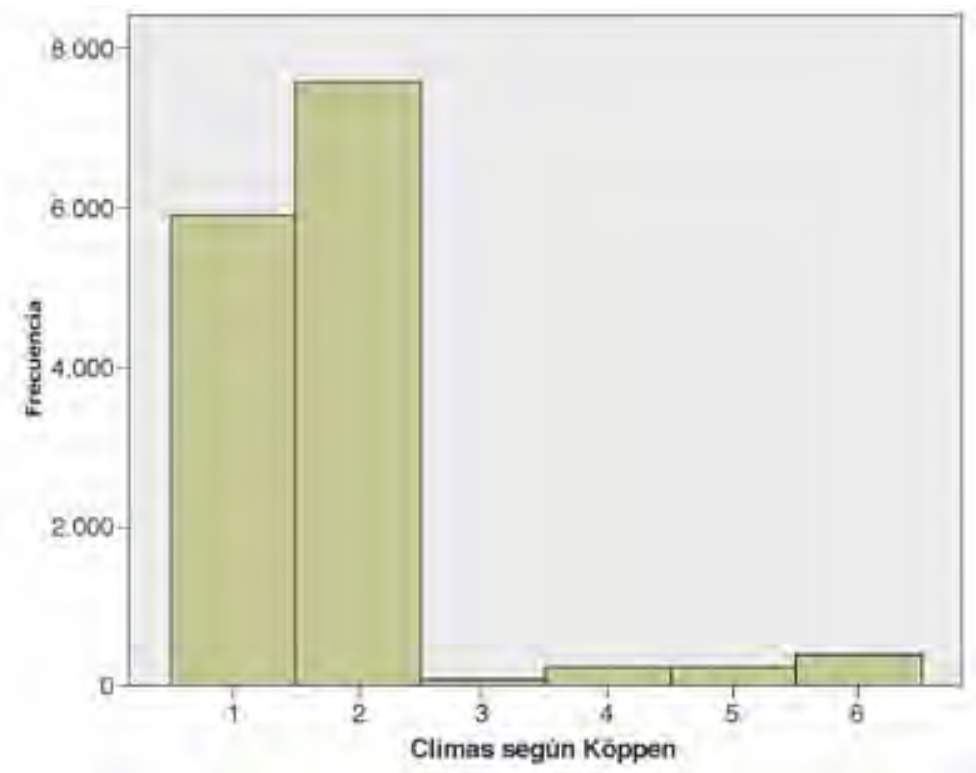

Figura 5. Gráfico de frecuencias de las extracciones según clima. $1=$ Ecuatorial, Monsonal $(A m) ; 2=$ Ecuatorial, invierno seco (Aw); 3= Estepa Fría (BSk); 4= Temperatura templada Humedad elevada - Verano caluroso (Cfa); $5=$ Temperatura templada - Invierno seco - Verano caluroso $(\mathrm{Cwa}) ; 6=$ Temperatura templada - Invierno seco - Verano templado $(\mathrm{Cwb})$ 


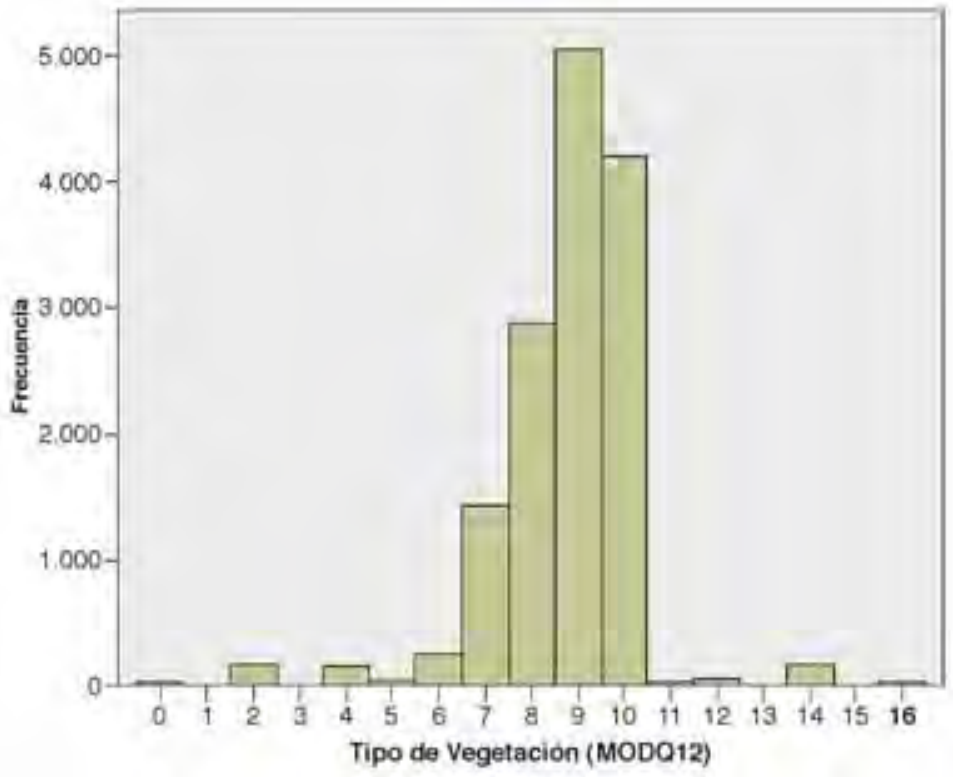

Figura 6. Gráfico de frecuencias de las extracciones según tipo de vegetación. $0=$ Agua; 1 = Coníferas perennes; 2 = Frondosas perennes; 4 = Frondosas caducas; $5=$ Bosques mixtos; $6=$ Matorral cerrado; $7=$ Matorral abierto; $8=$ Sabana arbolada; $9=$ Sabana $; 10=$ Pastizales; 11 = Humedales; 12 = Cultivos; $13=$ Urbano; $14=$ Mosaico de cultivos; $15=$ Hielo y Nieve; $16=$ Desnudo o escaso de vegetación

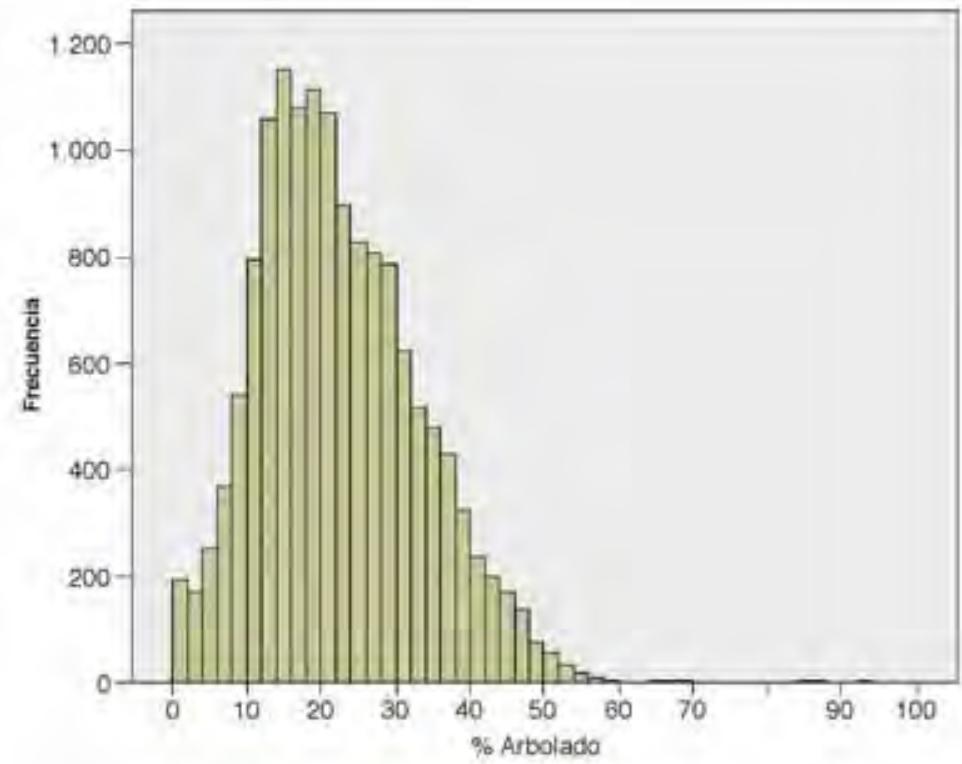

Figura 7. Gráfico de frecuencias de las extracciones según porcentaje de arbolado 


\subsection{Análisis de comparación de medias}

A continuación se ha realizado un test de comparación de medias del tipo ANOVA para los índices NBR y BAIn, utilizando como factor de análisis las tres variables auxiliares.

\subsubsection{Análisis según variable climática (Köppen)}

En la Figura 8 se aprecia que los climas ecuatoriales (Am y Aw) presentan valores de NBR mayores; en cambio, los climas de temperaturas templadas muestran valores más bajos. El caso del BAIn es similar, aunque aquí el clima Aw es el que muestra los valores más altos. Como resultado del test ANOVA (Tabla 3) el estadístico F mostró un valor más alto para BAIm indicando un mayor nivel de separabilidad entre los grupos.
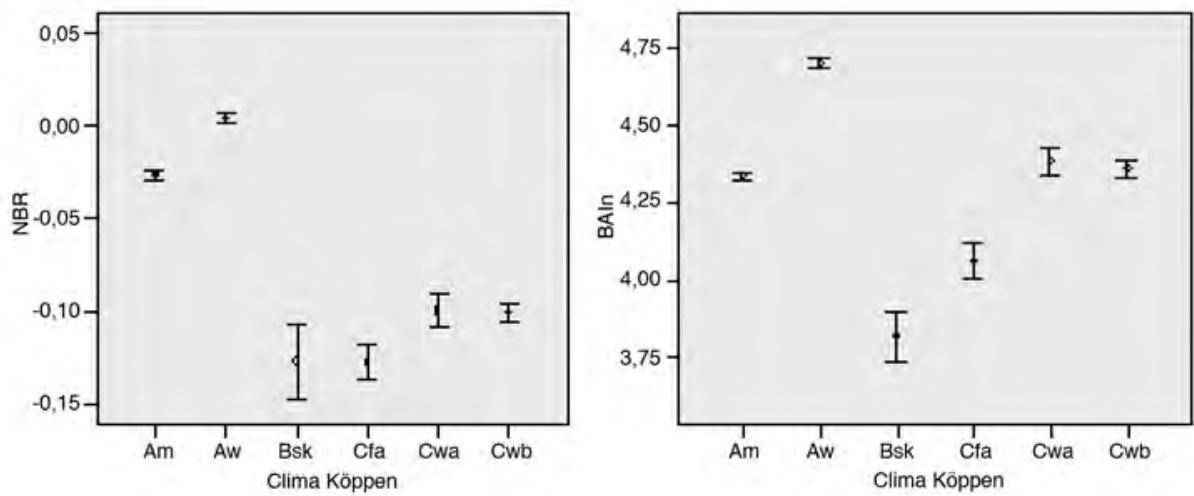

Figura 8. Gráfico de comparación de medias para NBR y BAIn según clima

Tabla 3. Test de ANOVA

\begin{tabular}{|c|c|c|r|r|r|c|}
\cline { 2 - 7 } \multicolumn{2}{c|}{} & $\begin{array}{c}\text { Suma de } \\
\text { cuadrados }\end{array}$ & \multicolumn{1}{c|}{ gl } & $\begin{array}{c}\text { Media } \\
\text { cuadrática }\end{array}$ & F & Sig. \\
\hline \multirow{2}{*}{ NBR } & Inter-grupos & 12,380 & 5 & 2,476 & 176,417 & 0,000 \\
\cline { 2 - 7 } & Intra-grupos & 202,988 & 14463 & 0,014 & & \\
\cline { 2 - 8 } & Total & 215,368 & 14468 & & & \\
\hline \multirow{2}{*}{ BAIn } & Inter-grupos & 557,175 & 5 & 111,435 & 366,312 & 0,000 \\
\cline { 2 - 8 } & Intra-grupos & 4399,751 & 14463 & 0,304 & & \\
\cline { 2 - 8 } & Total & 4956,926 & 14468 & & & \\
\hline
\end{tabular}




\subsubsection{Análisis según variable de vegetación (MODQ12)}

En la Tabla 4 se observan los resultados del test de comparación de medias para los índices agrupados según tipos de vegetación. El estadístico F presenta valores muy similares en ambos casos.

Tabla 4. Test de ANOVA

\begin{tabular}{|c|c|c|c|c|c|c|}
\cline { 2 - 7 } \multicolumn{2}{c|}{} & $\begin{array}{c}\text { Suma de } \\
\text { cuadrados }\end{array}$ & gl & $\begin{array}{c}\text { Media } \\
\text { cuadrática }\end{array}$ & F & Sig. \\
\hline \multirow{2}{*}{ NBR } & Inter-grupos & 10,653 & 13 & 0,819 & 57,865 & 0,000 \\
\cline { 2 - 7 } & Intra-grupos & 204,714 & 14455 & 0,014 & & \\
\cline { 2 - 7 } & Total & 215,368 & 14468 & & & \\
\hline \multirow{2}{*}{ BAIn } & Inter-grupos & 486,707 & 13 & 37,439 & 121,064 & 0,000 \\
\cline { 2 - 8 } & Intra-grupos & 4470,219 & 14455 &, 309 & & \\
\cline { 2 - 8 } & Total & 4956,926 & 14468 & & & \\
\hline
\end{tabular}

En la Figura 9 no se aprecia una diferenciación clara entre los tipos de vegetación quemada, por lo que se optó por agruparlos en clases más generales.
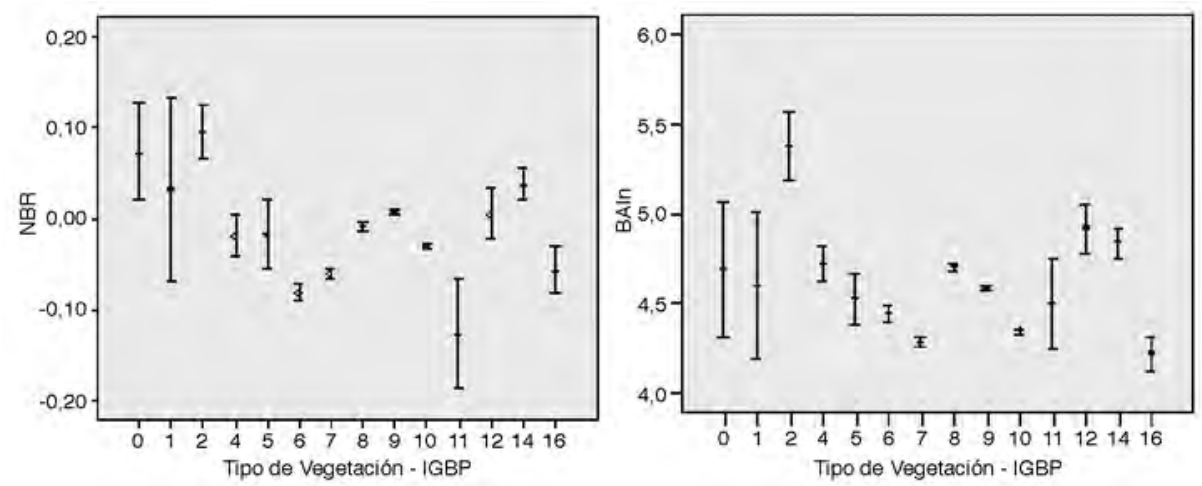

Figura 9. Gráfico de comparación de medias para NBR y BAI según tipo de vegetación

\subsection{Análisis según variable de vegetación (MODQ12) reclasificada}

Con la variable de tipo de vegetación agrupada en tres clases más generales podemos distinguir que los valores más altos, tanto en NBR como en BAIn, se encuentran en la clase de arbustivas. Las otras dos clases presentan valores menores. De igual forma que antes de la reagrupación de la variable, el estadístico F es mayor para el BAI que para el NBR. 
Tabla 5. Test de ANOVA

\begin{tabular}{|c|c|c|c|c|c|c|}
\cline { 2 - 7 } \multicolumn{2}{c|}{} & $\begin{array}{c}\text { Suma de } \\
\text { cuadrados }\end{array}$ & gl & $\begin{array}{c}\text { Media } \\
\text { cuadrática }\end{array}$ & F & Sig. \\
\hline \multirow{2}{*}{ NBR } & Inter-grupos & 4,085 & 4 & 1,021 & 69,917 & 0,000 \\
\cline { 2 - 7 } & Intra-grupos & 211,283 & 14464 & 0,015 & & \\
\cline { 2 - 7 } & Total & 215,368 & 14468 & & & \\
\hline \multirow{2}{*}{ BAIn } & Inter-grupos & 136,360 & 4 & 34,090 & 102,286 & 0,000 \\
\cline { 2 - 8 } & Intra-grupos & 4820,566 & 14464 & 0,333 & & \\
\cline { 2 - 8 } & Total & 4956,926 & 14468 & & & \\
\hline
\end{tabular}
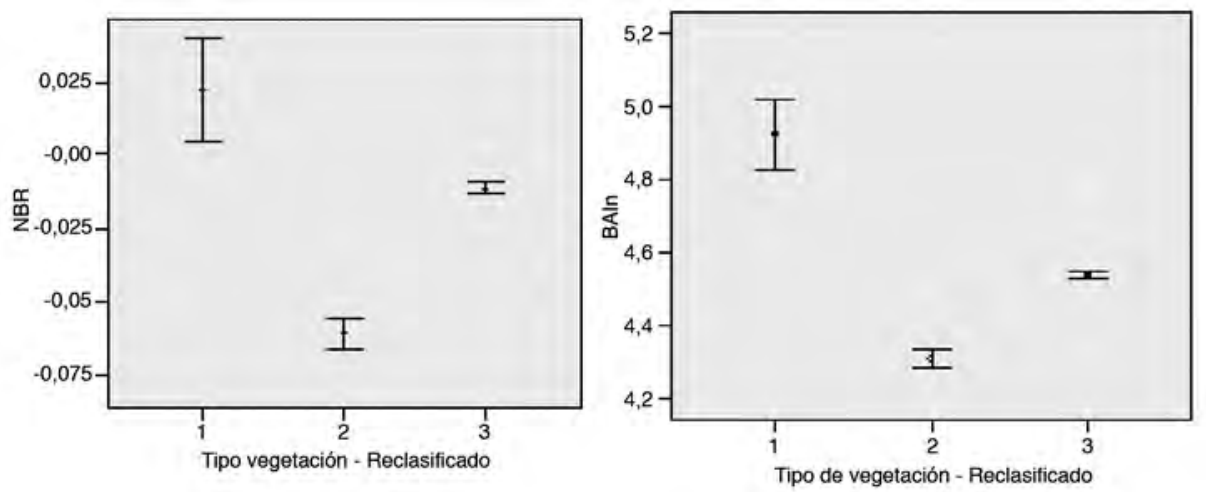

Figura 10. Gráfico de comparación de medias para NBR y BAI según tipo de vegetación reclasificado. $1=$ Arbustivas, $2=$ Matorrales y $3=$ Pastizales

\subsection{Análisis según variable de porcentaje de arbolado}

Dado que la variable de porcentaje de arbolado es continua se optó por reclasificarla; para ello se determinó el punto de corte de los grupos usando el percentil $10 \%$. Los grupos fueron los mostrados en la Tabla 6.

En este caso (Figura 11) podemos apreciar un claro aumento de los valores de los índices a medida que el porcentaje de arbolado aumenta, tanto para el caso del NBR como para BAIn. 
Tabla 6. Reclasificación de porcentaje de arbolado

\begin{tabular}{|c|c|}
\hline Grupo & Porcentaje de Arbolado \\
\hline 1 & $0-9$ \\
2 & $9-13$ \\
3 & $13-15$ \\
4 & $15-18$ \\
5 & $18-21$ \\
6 & $21-24$ \\
7 & $24-27$ \\
8 & $27-31$ \\
9 & $31-37$ \\
10 & $37-100$ \\
\hline
\end{tabular}

Tabla 7. Test de ANOVA

\begin{tabular}{|c|c|c|r|r|r|c|}
\cline { 2 - 7 } \multicolumn{2}{c|}{} & $\begin{array}{c}\text { Suma de } \\
\text { cuadrados }\end{array}$ & gl & $\begin{array}{c}\text { Media } \\
\text { cuadrática }\end{array}$ & F & Sig. \\
\hline \multirow{3}{*}{ NBR } & Inter-grupos & 6,295 & 9 & 0,699 & 48,371 & 0,000 \\
\cline { 2 - 7 } & Intra-grupos & 209,073 & 14459 & 0,014 & & \\
\cline { 2 - 8 } & Total & 215,368 & 14468 & & & \\
\hline \multirow{2}{*}{ BAIn } & Inter-grupos & 169,986 & 9 & 18,887 & 57,049 & 0,000 \\
\cline { 2 - 8 } & Intra-grupos & 4786,940 & 14459 &, 331 & & \\
\cline { 2 - 8 } & Total & 4956,926 & 14468 & & & \\
\hline
\end{tabular}
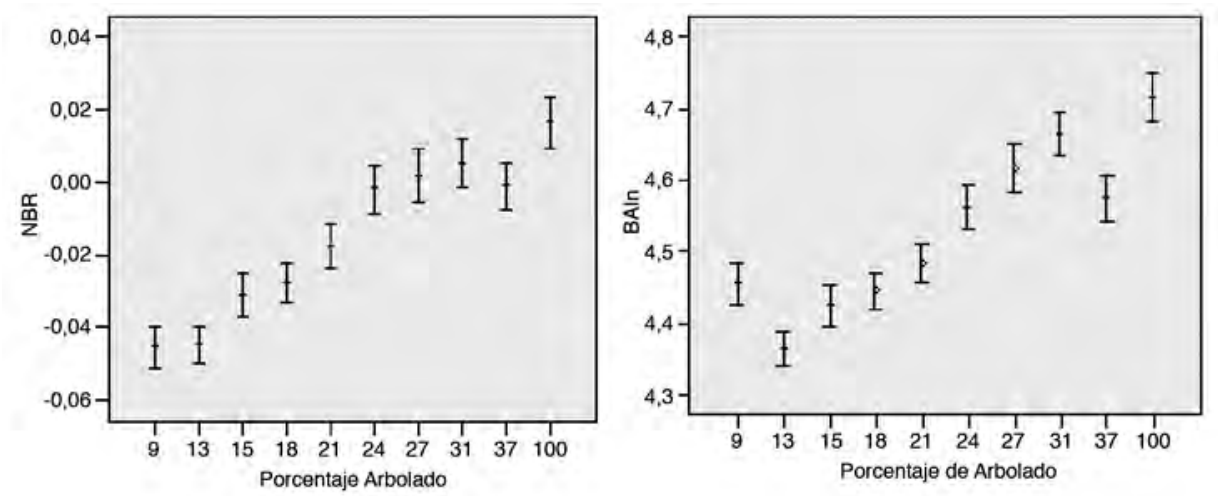

Figura 11. Gráfico de comparación de medias para NBR y BAI según porcentaje de arbolado 


\subsection{Análisis según la variable reclasificada de porcentaje de arbolado}

Para disminuir la confusión que presentaron algunas de las clases en que se agrupo la variable de arbolado, hemos propuesto una nueva agrupación en cuatro grupos (Tabla 8), observándose ahora una separación mucho más marcada entre los nuevos grupos.

Tabla 8. Reclasificación de porcentaje de arbolado

\begin{tabular}{|c|c|}
\hline Grupo & Porcentaje de Arbolado \\
\hline 1 & $0-13$ \\
2 & $13-21$ \\
3 & $21-37$ \\
4 & $37-100$ \\
\hline
\end{tabular}

Tabla 9. Test de ANOVA

\begin{tabular}{|c|c|c|r|r|r|c|}
\cline { 2 - 7 } \multicolumn{2}{c|}{} & $\begin{array}{c}\text { Suma de } \\
\text { cuadrados }\end{array}$ & gl & $\begin{array}{c}\text { Media } \\
\text { cuadrática }\end{array}$ & F & Sig. \\
\hline \multirow{2}{*}{ NBR } & Inter-grupos & 6,108 & 3 & 2,036 & 140,736 & 0,000 \\
\cline { 2 - 7 } & Intra-grupos & 209,260 & 14465 & 0,014 & & \\
\cline { 2 - 7 } & Total & 215,368 & 14468 & & & \\
\hline \multirow{2}{*}{ BAIn } & Inter-grupos & 151,740 & 3 & 50,580 & 152,260 & 0,000 \\
\cline { 2 - 8 } & Intra-grupos & 4805,186 & 14465 & 0,332 & & \\
\cline { 2 - 8 } & Total & 4956,926 & 14468 & & & \\
\hline
\end{tabular}
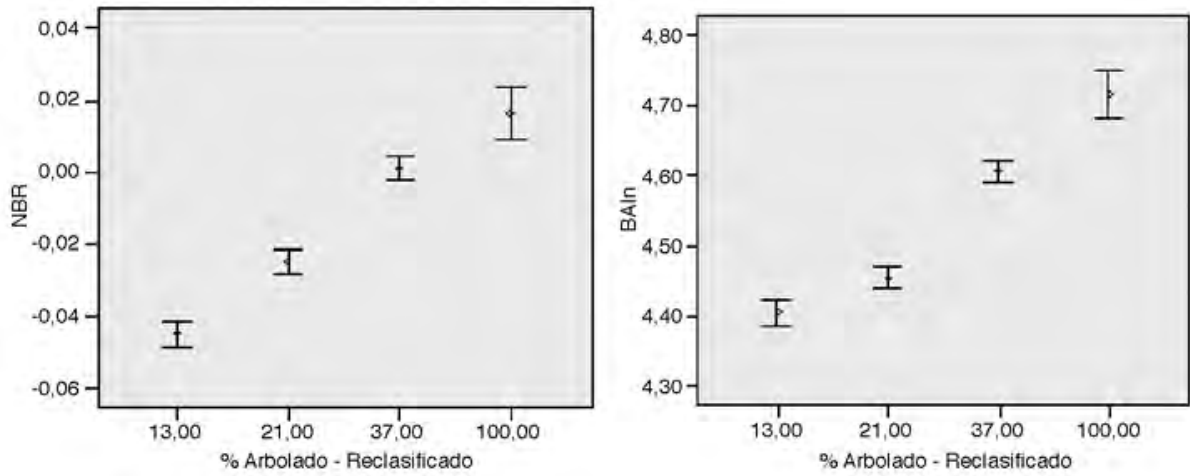

Figura 12. Gráfico de comparación de medias para NBR y BAI según porcentaje de arbolado 
Se puede ver que a medida que el porcentaje de arbolado es mayor los índices, tanto NBR como BAIn, tienden a mostrar valores más altos.

\section{Discusión de los resultados}

Si analizamos el comportamiento del histograma de frecuencias del NBR observamos que se ajusta bastante bien a una curva normal, ya que este índice se ha diseñado de esta forma. El caso del BAI es distinto; al no ser un índice normalizado, fue necesario aplicar una transformación que permitiera normalizar la variable y reducir por tanto la amplitud en la desviación típica que presenta originalmente. El amplio rango de valores que indican los índices nos muestra las diferentes características que presentan las áreas quemadas en las distintas regiones, estas diferencias suelen estar asociadas principalmente a las características del combustible, que en adición a otros factores determinan la severidad y por tanto la intensidad y persistencia de la señal quemada.

Otro factor que se debe tener en cuenta es el desfase temporal entre los perímetros quemados de referencia (Landsat - CBERS) y las imágenes correspondientes al muestreo que proviene de compuestos MODIS de 16 días; esto puede llevar a la pérdida de la señal quemada producto de la regeneración de la vegetación, especialmente en regiones de alta actividad productiva como son las zonas tropicales.

Los climas de zonas ecuatoriales (Aw, Am) presentan la mayor concentración de muestras. Esto se debe a que precisamente estos climas presentan características ecológicas que favorecen la propagación de los incendios; por lo demás, las zonas muestreadas en estas regiones coinciden con áreas donde el fuego es utilizado como herramienta en labores agroforestales, que en muchas ocasiones escapa del control y afecta grandes extensiones.

En cuanto a los tipos de vegetación se observa que la concentración más alta de muestras se ha realizado en pastizales y matorrales abiertos; en estas zonas, normalmente, la propagación de los incendios es más rápida y suele afectar a extensiones mayores que en zonas arboladas; este hecho es importante ya que estas zonas presentan una capacidad de regeneración rápida, disminuyendo la permanencia de la señal quemada. Del mismo modo, la variable de arbolado muestra que prácticamente no se han realizado extracciones en zonas con valores de arbolado mayores al 50\%; esto tiene relación con lo mencionado anteriormente, siendo las zonas de componente arbolado inferior donde los incendios afectan a mayores superficies.

El tipo de vegetación arbustiva (Figura 10) presenta los valores más altos de NBR y BAIn; del mismo modo, el porcentaje de arbolado (Figura 12) viene a confirmar esto mostrando una relación entre el valor de los índices y la proporción de arbolado, a medida que aumenta el arbolado también lo hacen los valores de los índices. Esta tendencia se puede explicar dado que las formaciones con mayor proporción de árboles tienen mayor biomasa y por tanto más combustible, lo que se traduce en mayor señal de carbón. Este punto es especialmente importante a la hora de establecer criterios para la definición de umbrales, siendo posible diferenciar los umbrales en zonas según tengan más o menos combustible disponible. 


\section{Conclusiones}

Los índices utilizados en este estudio tienden a seguir el mismo comportamiento, cabe notar que la separabilidad entre grupos del BAI mejoró considerablemente una vez realizada la normalización, obteniéndose resultados similares en todos los test de ANOVA realizados.

Las variables de vegetación (tipo de vegetación y porcentaje de arbolado) parecen explicar de mejor forma las variaciones que sufren los índices en las distintas regiones; el factor crítico donde se diferencian en mayor medida los valores de los índices tiene relación con el arbolado.

Sería interesante analizar la relación conjunta entre el porcentaje de arbolado y la variable climática, lo que permitiría conocer si este comportamiento tiene variaciones en las diferentes regiones.

\section{Referencias bibliográficas}

Barbosa, P.M., Grégoire, J.M. y Pereira, J.M.C. (1999). An algorithm for extracting burned areas from time series of AVHRR GAC data applied at a continental scale. Remote Sensing of Environment 69: 253-263.

Beck, C., Grieser, J., Kottek, M., Rubely, F. y Rudolf, B. (2006). Characterizing global climate change by means of Köppen climate classification. The Global Precipitation Climatology Centre (GPCC), Alemania.

Cocke, A.E., Fule, P.Z. y Crouse, J.E. (2005). Comparison of burn severity assessments using Differenced Normalized Burn Ratio and ground data. International Journal of Wildland Fire 14: 189-198.

Chuvieco, E., Martín, M.P. y Palacios, A. (2002). Assessment of different spectral indices in the red-near-infrared spectral domain for burned land discrimination. International Journal of Remote Sensing 23 (23): 5103-5110.

Chuvieco, E., Opazo, S., Sione, W., Del Valle, H., Anaya, J., Di Bella, C., Cruz, I., Manzo, L., López, G., Mari, N., González-Alonso, F., Morelli, F., Setzer, A., Csiszar, I., Karpandegui, J., BastarriKa, A. y Libonati, R. (2007). Global Burned Land Estimation in Latin America using MODIS Composite Data. Ecological Applications: en prensa.

FAO (2006). Evaluación de los Recursos Forestales Mundiales 2005. Organización de las naciones unidas para la agricultura y la alimentación, Roma, 351pp.

Heredia, A., Martínez, S., Quintero, E., Piñeros, W. y Chuvieco, E. (2003). Comparación de Distintas Técnicas de Análisis Digital para la Cartografía de Áreas Quemadas con Imágenes Landsat ETM+. GeoFocus 3: 216-234. 
Justice, C.O., Giglio, L., Korontzi, S., Owens, J., Morisette, J.T., Roy, D., Descloitres, J., Alleaume, S., Petitcolin, F. y Kaufman, Y. (2002). The MODIS fire products. Remote Sensing of Environment 83(1-2): 244-262.

Key, C. y Benson, N. (1999). The Normalized Burned Ratio, a Landsat TM radiometric index of burn severity incorporating multi-temporal differencing. Disponible en http://www.nrmsc.usgs.gov/ research/ndbr.htm

LEVINE, J.S. (1996). Biomass burning and global change. MIT, Cambridge, Mass.

Loboda, T., O’NeALy, K.J. y Csiszar, I. (2007). Regionally adaptable dNBR-based algorithm for burned area mapping from MODIS data. Remote Sensing of Environment, en prensa.

Martín, M.P. (1998). Cartografía e inventario de incendios forestales en la Península Ibérica a partir de imágenes NOAA-AVHRR. Tesis Doctoral. Departamento de Geografía, Universidad de Alcalá, Alcalá de Henares.

Martín, M.P., Díaz-Delgado, R., Chuvieco, E. y Ventura, G. (2002). Burned land mapping using NOAA-AVHRR and TERRA-MODIS. En IV International Conference on Forest Fire Research. 2002 Wildland Fire Safety Summit. Millpress, Luso, Coimbra, Portugal.

Miller, J.D. y Thode, A.E. (2007). Quantifying burn severity in a heterogeneous landscape with a relative version of the delta Normalized Burn Ratio (dNBR). Remote Sensing of Environment, en prensa.

Moreno, J.M. (1998). Large Forest Fires. Backhuys Publisher, Leiden (The Netherlands).

Parisien, M.A., Vernon, S.P, Yonghe, J.M., Bosch, E.M. y Stocks, B.J. (2006). Spatial Patterns of forest fires in Canada, 1980-1999. International Journal of Wildland fire 15: 361-374.

PereIRA, J.M. (1999). A Comparative Evaluation of NOAA/AVHRR Vegetation Indexes for Burned Surface Detection and Mapping. IEEE Transactions on Geoscience and Remote Sensing 37(1): 217-226.

Puigdefábregas, J. y Mendizábal, T. (1998). Perspectives on desertification: western Mediterranean. Journal of Arid Environment 39: 209-224.

Roy, D., Jin, Y., Lewis, P. y Justice, C. (2005). Prototyping a global algorithm for systematic fire-affected area mapping using MODIS time series data. Remote Sensing of Environment 97: 137-162.

Sifakis, N., PARONIS, D. y Keramitsoglou, I. (2004). Combining AVHRR imagery with CORINE Land Cover data to observe forest fires and to assess their consequences. International Journal of Applied Earth Observation and Geoinformation 5: 263-274.

Simon, M., Plummer, S., Fierens, F., Hoelzemann, J.J. y Arino, O. (2004). Burnt area detection at global scale using ATSR-2: The GLOBSCAR products and their qualification. Journal of Geophysical Research - Atmospheres 109, D14S02, doi: 10.1029/2002JD003622: 1-16. 
SOKAL, R. y RHOLF, F. (Eds.) (1995). Biometry, The principles and practice of statistics in biology research. W.H. Freeman, New York.

Tansey, K., Grégoire, J.M., Binaghi, E., Boschetti, L., Brivio, P.A., Ershov, D., Flasse, E., Fraser, R., Graetz, D., Maggi, M., Peduzzi, P., Pereira, J., Silva, J., Sousa, A. y Stroppiana, D. (2004). A Global Inventory of Burned Areas at $1 \mathrm{Km}$. Resolution for the year 2000 derived from SPOT VEGETATION Data. Climatic Change 67: 345-377.

Tansey, K., Grégoire, J.M., Stroppiana, D., Sousa, A., Silva, J., Pereira, J.M., Boschetti, L., Maggi, M., Brivio, P.A., Fraser, R., Flasse, S., Ershov, D., Binaghi, E., Graetz, D. y Peduzzi, P. (2004). Vegetation burning in the year 2000: Global burned area estimates from SPOT VEGETATION data. Journal of Geophysical Research - Atmospheres 109, D14S03, doi: 10.1029/2002JD003598: 2-22.

TrigG, S. y Flasse, S. (2001). An evaluation of different bi-spectral spaces for discriminating burned shrub-savannah. International Journal of Remote Sensing 22(13): 2641-2647.

Van der Werf, G.R., Randerson, J.T., Collatz, G.J., Giglio, L., Kasibhatla, P.S., Arellano, A.F., Olsen, S.C. y KasischKe, E.S. (2004). Continental Scale-partitioning of fire emissions during the 1997 to 2001 El Niño/La Niña period. Science 303: 73-76. 